\title{
Nonorthogonal Multiple Access for 5G and Beyond
}

\author{
Oğuz Kucur $\mathbb{D}$, ${ }^{1}$ Güneş Karabulut Kurt $\mathbb{D}{ }^{2}$ \\ Muhammad Zeeshan Shakir, ${ }^{3}$ and Imran Shafique Ansari ${ }^{4}$ \\ ${ }^{1}$ Gebze Technical University, Kocaeli, Turkey \\ ${ }^{2}$ Istanbul Technical University, Istanbul, Turkey \\ ${ }^{3}$ University of the West of Scotland, Paisley, UK \\ ${ }^{4}$ Global College of Engineering and Technology in Partnership with UWE Bristol, Muscat, Oman
}

Correspondence should be addressed to Oğuz Kucur; okucur@gtu.edu.tr

Received 3 May 2018; Accepted 3 May 2018; Published 5 July 2018

Copyright (c) 2018 Oğuz Kucur et al. This is an open access article distributed under the Creative Commons Attribution License, which permits unrestricted use, distribution, and reproduction in any medium, provided the original work is properly cited.

Over the past decades, rapid developments and evolving demands of wireless communications changed the selected multiple access (MA) technique in each generation. Today, increasing demands of high spectral/energy efficiency, high connectivity, and low latency of future generations such as $5 \mathrm{G}$ and beyond can be satisfied by Non-orthogonal Multiple Access (NOMA). Different from conventional MA techniques such as frequency division MA, time division MA, code division MA, and orthogonal frequency division MA of previous generations, which are based on orthogonal resources, the key idea in NOMA is to allocate non-orthogonal resources to serve multiple users, yielding a high spectral efficiency while allowing some degree of interference at receivers. Recently proposed NOMA techniques can be mainly categorized into two groups: power domain and code domain. In power domain NOMA, multiple users are superposed by different power levels opposite to the channel conditions such that successive interference cancellation is applied at receivers, providing a good tradeoff between system throughput and user fairness. Other NOMA approaches include low density spreading (LDS), sparse code multiple access (SCMA), multi-user shared access (MUSA), and interleave division multiple access (IDMA), and so on.

Despite having several advantages for $5 \mathrm{G}$ and beyond, challenges and obstacles exist in the efficient deployment of NOMA. The motivation behind this special issue has been to address such challenging issues of NOMA. Following a rigorous review process (including a second review round), 7 outstanding papers have been finally selected for inclusion in the special issue. The accepted papers cover a wide range of research subjects in the broader area of NOMA to meet the increasing demands of $5 \mathrm{G}$ and beyond.

The paper entitled "A Tutorial on Non-Orthogonal Multiple Access (NOMA) for 5G and Beyond" by M. Aldababsa et al. provides a unified model for NOMA, including uplink and downlink transmissions, along with the extensions to multiple input multiple output and cooperative communication scenarios. The authors compare the performances of orthogonal multiple access (OMA) and NOMA networks through numerical examples and also provide discussions about implementation aspects and open issues.

The paper "NOMA for Multinumerology OFDM Systems" by A. T. Abusabah et al. proposes an orthogonal frequency division multiplexing (OFDM) based NOMA scheme, which utilizes the multi-numerology concept, i.e., different subcarrier spacings to reduce the constraints associated with the multi-user detection. By this scheme, which is less spectrally efficient than conventional NOMA schemes, but more spectrally efficient than OMA schemes, the authors improve both user fairness and error performance.

A new member of NOMA family, named as non-uniform code multiple access (NCMA), is introduced in "Nonuniform Code Multiple Access", by C. Yan et al. In NCMA, different transmitted layers can be generated from different complex multi-dimensional constellations. Benefiting from the proposed codebook design, the minimum intra-partition distance can be increased. Simulation results demonstrate performance improvement against SCMA.

The paper "Weighted Proportional Fair Scheduling for Downlink Nonorthogonal Multiple Access" by M.-R. Hojeij 
et al. has presented a novel weighted proportional fair scheduling scheme for NOMA. The proposed design can adapt the weights and maximize the capacity while improving the long term fairness amongst the users. The study has been supported by the set of the simulation results and comparative evaluation with the benchmarks such as OMA and classic NOMA-based proportional fair scheduler. The concept has potential to be exploited further for multi-user and multiantenna scenarios with critical focus on the complexity of the design.

The paper, "Multiway Physical-Layer Network Coding via Uniquely Decodable Codes" by M. Kulhandjian et al. has proposed a novel but simple network coding by exploiting Uniquely Decodable (UD) Codes to allow users to uniquely recover the information bits from the noise channel. The proposed decoder has been simulated and compared with the traditional maximum likelihood (ML) decoders in terms of the bit error rate and sum rate. It has been shown that the proposed scheme utilizing UD codes achieve near-ML performance with less complex design and improve the sum rates almost 7 and 16 times compared to traditional physical layer network coding scheme.

The paper "An Efficient SCMA Codebook Optimization Algorithm Based on Mutual Information Maximization" by C. Dong et al. proposes an efficient SCMA codebook optimization algorithm to maximize mutual information between the discrete input and continuous output. Initially, SCMA signal model is described according to superposition modulation structure wherein the channel matrix is column extended that can well represent the relationship between the codebook matrix and received signal. The superposition model can well describe the relationship between the codebook matrix and received signal. Based on this superposition model, an iterative codebook optimization algorithm is proposed wherein the linear search method is applied in order to find locally optimal codebooks thereby maximizing mutual information between discrete input and continuous output. This algorithm can efficiently adapt to multi-user channels with arbitrary channel coefficients. The simulation results demonstrate that the proposed algorithm approaches Gaussian capacity upper bound in low and medium signal-to-noise ratio regimes. Moreover, the performance loss in non-additive white Gaussian noise channel is relatively small when compared with the upper bound. In addition, message passing algorithm works well with the codebook optimized with the proposed algorithm.

The paper "On the Performance of Security-Based Nonorthogonal Multiple Access in Coordinated Multipoint Networks" by Y. Tian et al. focuses on the security-based NOMA (S-NOMA) systems that aim to improve the physical layer security issues of conventional NOMA systems in the coordinated multi-point (CoMP) networks. The authors analyze the secrecy performance of S-NOMA in CoMP, i.e., the secrecy sum-rate and the secrecy outage probability, and demonstrate that the proposed S-NOMA outperforms conventional NOMA in terms of the secrecy outage probability and security-based effective sum-rate, especially when the target transmission data rate is high.

\section{Acknowledgments}

We would like to thank all the authors who submitted their excellent research articles to this special issue and all the reviewers for providing their valuable and timely feedback through the review process, which helped to improve the quality of this special issue.

Oğuz Kucur Güneş Karabulut Kurt Muhammad Zeeshan Shakir Imran Shafique Ansari 


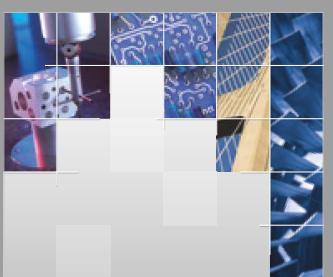

\section{Enfincering}
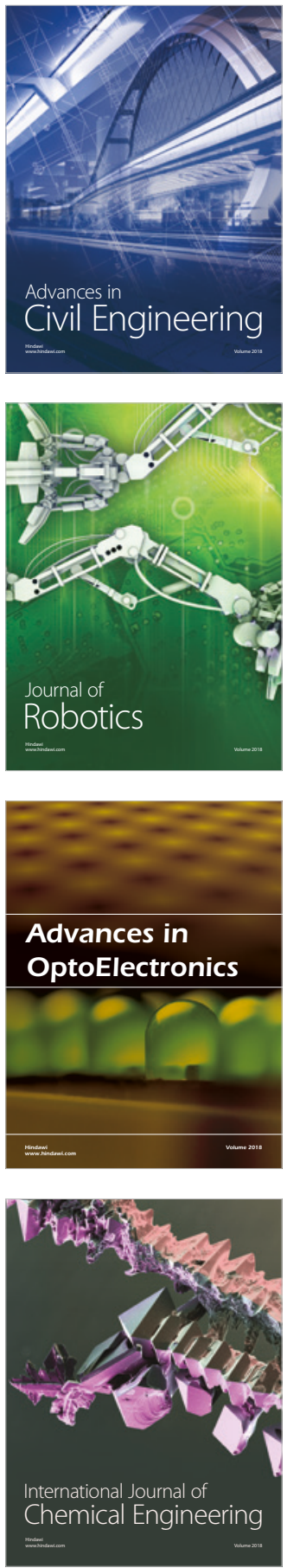

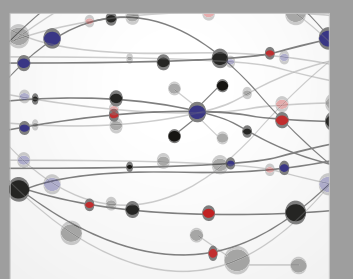

\section{Rotating \\ Machinery}

The Scientific World Journal

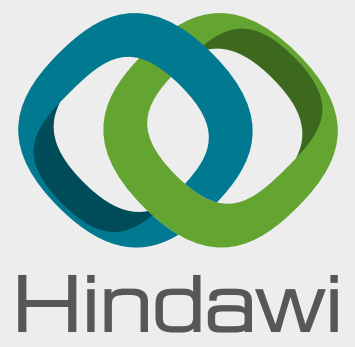

Submit your manuscripts at

www.hindawi.com
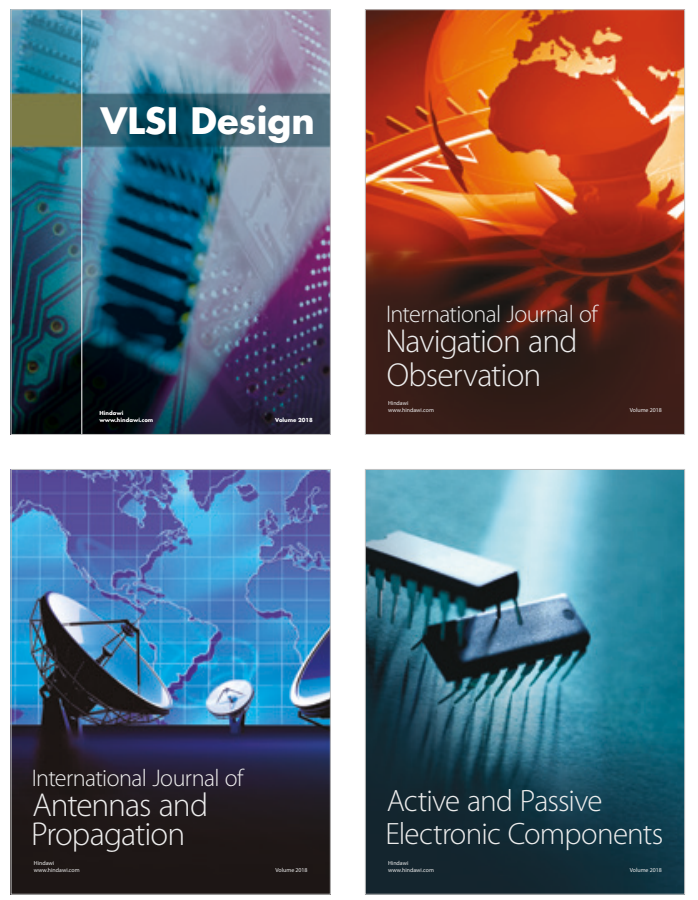
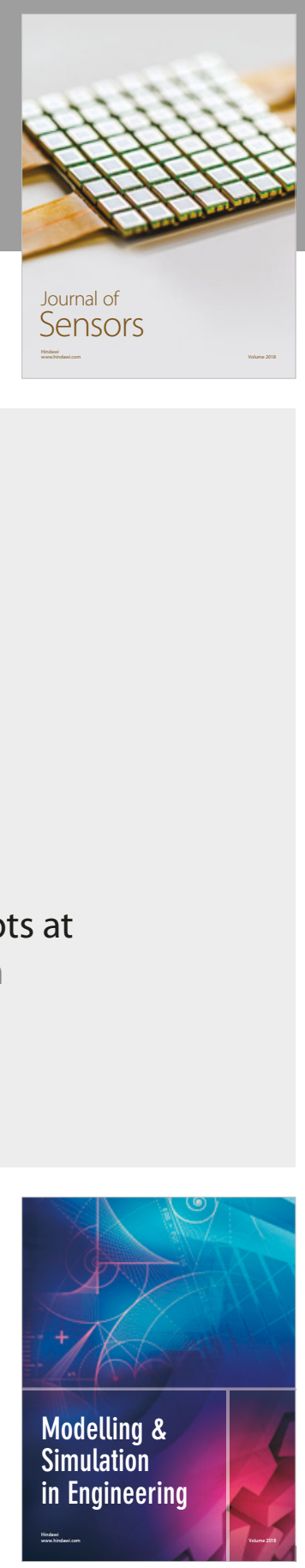

\section{Advances \\ Multimedia}
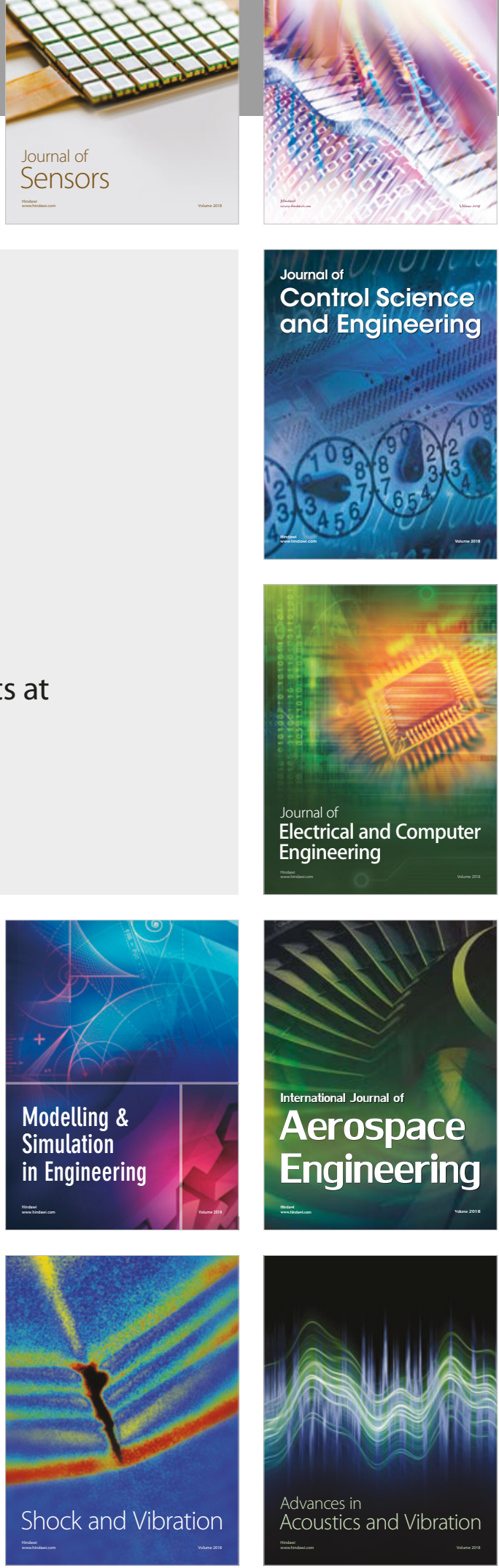